\title{
Immunotherapy-responsive gastric cancers identified
}

The anti-PD-1 antibody pembrolizumab is an FDA-approved treatment of PD-L1-positive metastatic gastric cancer; however, only a subset of patients respond to this agent. Now, data from a phase II trial shed some light on the reasons for these variable responses.

"We designed a phase II trial, with integrated genomic analysis of all baseline tumour tissue samples and genomic profiling of circulating tumour DNA (ctDNA) samples in order to understand the disease characteristics of responders and nonresponders," explains senior investigator Jyun Lee. This openlabel trial included 61 patients with metastatic and/or recurrent gastric adenocarcinomas. The cohort included six patients with EpsteinBarr virus (EBV)-positive disease and seven with microsatellite-

instability high (MSI-H) disease.
Similar to the findings of previous trials, an overall response rate (ORR) of $24.6 \%$ was observed. Notably, $85.7 \%$ of patients with MSI-H tumours, and all patients with EBV-positive disease, responded to pembrolizumab.

Researchers also investigated the relationship between biopsybased tumour mutational burden (TMB) and response, observing a positive correlation with response to pembrolizumab (area under the curve $0.74 ; P=0.006$ ). A similar relationship was observed between ctDNA-based TMB, quantified using a 73-gene next-generation sequencing panel, and response to pembrolizumab. In a longitudinal analysis of samples from a subset of patients, changes in ctDNA level at 6 weeks after treatment with pembrolizumab were found to be predictive of a response:

\section{LIVER DISEASE}

\section{Senescence prevents regeneration after acute liver injury}

Acute severe liver injury impairs hepatocyte regeneration through the induction of senescence, according to new research. Strikingly, senescence was found to spread between neighbouring hepatocytes through paracrine TGF $\beta$ signalling.

The liver has a remarkable capacity to regenerate after moderate damage. However, liver regeneration can fail after severe insults, such as acute paracetamolrelated injury. "It was apparent for some patients requiring liver transplantation for sudden forms of liver failure that their livers were unable to regenerate despite the raw material for regeneration still being present," explains author Thomas Bird. "We asked why regeneration fails in these otherwise regenerative hepatocytes, even after the toxins causing damage are no longer detectable."

As previous research had suggested that cellular senescence might occur after years of liver injury, Bird and colleagues assessed human liver specimens obtained from patients at the onset of severe acute injury. Hepatocytes from these previously healthy livers expressed high levels of senescence markers, and levels correlated with the extent of hepatic necrosis. These results were confirmed in paracetamol and carbon tetrachloride mouse models of acute liver injury. Notably, in senescence formation, improved hepatocyte regeneration, indicating that senescence impairs liver recovery. Using genetically engineered mouse models, the authors discovered that senescence spreads between adjacent cells via the secretion of paracrine factors; further functional studies revealed that macrophage-derived TGF $\beta$ was required for optimal senescence induction. "Most importantly, we showed, in proof-of-principle studies in mice, that targeting the TGF $\beta$ senescence pathway can lead to clinically meaningful improvements in liver regeneration, knockout of p21, a key protein involved all four patients with increased ctDNA levels at 6 weeks had disease progression within 100 days of starting treatment.

"The most significant finding of this work is that patients with MSI-Hor EBV-positive gastric cancer have robust and sustained responses to pembrolizumab monotherapy," summarizes Lee, adding, "we also demonstrated that ctDNA mutational burden correlates very well with TMB and that decreasing ctDNA levels are a statistically significant predictor of prolonged progression-free survival." Prospective validation of these findings, which have the potential to substantially improve the treatment of metastatic gastric cancer, is eagerly awaited.

Peter Sidaway, Nature Reviews Clinical Oncology This article is modified from the original in
Nat. Rev. Clin. Oncol. (https://doi.org/10.1038/s41571-018-0079-y).

ORIGINAL ARTICLE Kim, S. T. et al. Comprehensive molecular characterization of clinical responses to PD-1 inhibition in metastatic gastric cancer. Nat.Med. https://doi.org/10.1038/s41591-0180101-z (2018)

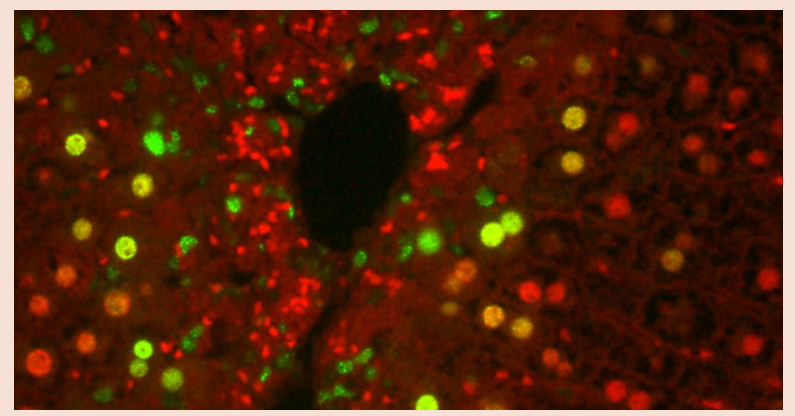

Hepatocytes (red) and markers of senescence (green) around area of liver damage. Image courtesy of T. Bird, University of Edinburgh, UK

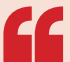

\section{targeting}

the TGF $\beta$

senescence

pathway

can lead to

clinically

meaningful

improvements

in liver

regeneration function and overall survival following paracetamol poisoning," says Bird.

Given the urgent need for effective therapies for acute liver failure, the investigators plan to move forward to clinical trials of TFG $\beta$ inhibitor therapy in this setting. "We have the advantage that other drugs in this class are already in phase I and phase II clinical oncology trials, so we hope to quickly move these treatments towards the clinic," Bird concludes.

Hugh Thomas

ORIGINAL ARTICLE Bird, T. G. et al. TGF $\beta$ inhibition restores a regenerative response in acute liver injury by suppressing paracrine senescence. Sci. Transl Med. 10, eaan1230 (2018) 